\title{
THE USE OF A NOVEL ALDEHYDE-FUNCTIONALIZED CHITOSAN HYDROGEL TO PREPARE POROUS TUBULAR SCAFFOLDS FOR VASCULAR TISSUE ENGINEERING APPLICATION
}

\author{
Eduardo P. Azevedo ${ }^{\mathrm{a}, *, \#}$ and Vijay Kumar ${ }^{\mathrm{b}}$ \\ ${ }^{a}$ Laureate International Universities, Universidade Potiguar, 59080-400 Natal - RN, Brazil \\ ${ }^{b}$ Department of Pharmaceutical Sciences and Experimental Therapeutics, The University of Iowa, 52242 Iowa City - IA, USA
}

Recebido em 20/02/2016; aceito em 12/05/2016; publicado na web em 15/07/2016

\begin{abstract}
THE USE OF A NOVEL ALDEHYDE-FUNCTIONALIZED CHITOSAN HYDROGEL TO PREPARE POROUS TUBULAR SCAFFOLDS FOR VASCULAR TISSUE ENGINEERING APPLICATIONS. In this work, porous tubular scaffolds were prepared from a novel water soluble aldehyde-functionalized chitosan (ALDCHIT) hydrogel, which was obtained by dissolving this chitosan derivative in water and using oxidized dextrose (OXDEXT) as the crosslinking agent at different ALDCHIT:OXDEXT mole ratios (10:1, 10:2 and 10:4). By increasing the amount of OXDEXT in respect to ALDCHIT the hydrogels became more rigid and could absorb more than $200 \%$ of its weight in water. Since the ALDCHIT:OXDEXT 10:4 was the most stable hydrogel, its ability to form porous tubular scaffolds was investigated. The tubular scaffolds were prepared by the lyophilization method, where the orientation of the pores was controlled by exposing either the internal or the external surface of the frozen hydrogel during the sublimation step. When only the inner surface of the frozen hydrogel was exposed, tubular scaffolds with a highly porous lumen and a sealed outer surface were obtained, where the orientation of the pores, their sizes and interconnectivity seem to be optimum for vascular tissue engineering application.
\end{abstract}

Keywords: chitosan derivative; hydrogels; water uptake capacity; rheological properties; tubular scaffolds.

\section{INTRODUCTON}

Chitosan has been extensively used to synthesize hydrogels due to its good crosslinking ability, which has been attributed to the presence of free amine groups in its backbone. ${ }^{1}$ In addition, its biodegradability, biocompatibility and non-toxicity make the chitosan hydrogels very attractive for biomedical applications. ${ }^{2}$ Chitosan hydrogels have been prepared as microspheres,,${ }^{3,4}$ nanospheres,${ }^{5}$ beads,${ }^{6-8}$ sponges, ${ }^{9}$ wound dressings, ${ }^{10}$ films ${ }^{11}$ and scaffolds for tissue engineering. ${ }^{12-14}$

These hydrogels are usually prepared through interchain interactions between the chitosan's free amine groups, which is achieved by using a crosslinking agent ${ }^{15}$ such as formaldehyde ${ }^{1}$ and glutaraldehyde. ${ }^{16,17}$ The disadvantage of these crosslinking agents, especially glutaraldehyde, is their toxicity to human tissues even at small traces, which has limited the use of chitosan hydrogels as scaffolds for tissue engineering. ${ }^{18,19}$ On the other hand, oxidized starch, ${ }^{20}$ oxidized dextran, ${ }^{16}$ oxidized sugars ${ }^{21}$ and genipin ${ }^{19,22}$ have been used as alternatives to gluteraldehyde and have exhibited minimal toxicity. ${ }^{18}$

Chemical modifications have been performed to prepare chitosan derivatives with enhanced biological and physicochemical properties. Recently, chitosans carrying aldehyde functions were successfully prepared by our group, ${ }^{23}$ where besides being water-soluble, this chitosan derivative could self-crosslink through Schiff base reaction forming semi-solid hydrogels. Furthermore, scanning electron microscope analysis of the lyophilized hydrogels showed a highly porous structure with interconnecting pores, which was evidenced by their high water uptake capacity (more than 400\%). ${ }^{24}$ Considering that the presence of interconnecting pores, an optimal pore size and shape, and the biocompatibility/biodegradability aspect are among the main conditions in which a scaffold for tissue engineering has to fulfill, ${ }^{16}$ we hypothesize that these hydrogels prepared from this novel water soluble aldehyde-functionalized chitosan has great potential for

*e-mail: azevedoep@hotmail.com

"Programa de Mestrado em Biotecnologia such application. Thus, the successful design of a hydrogel without the addition of a toxic crosslinker would enable the development of biocompatible scaffolds for tissue engineering applications.

In this study, porous tubular scaffolds were prepared from aldehyde-functionalized chitosan semi-solid hydrogels using the lyophilization method. Additional crosslinkage was achieved by using oxidized dextrose as the crosslinking agent. Furthermore, the influence of the amount of oxidized dextrose on the rheological properties, as well as on the water uptake capacity of these hydrogels, was assessed. Finally, the ability of this novel hydrogel to form porous tubular constructs was investigated for potential application as scaffolds for vascular tissue engineering, where the influence of some experimental parameters on the size and orientation of the pores was assessed.

\section{EXPERIMENTAL}

\section{Preparation of the aldehyde-functionalized chitosan (ALDCHIT)}

Chitosan (degree of deacetylation $87 \%$ as determined by ${ }^{1} \mathrm{H}-\mathrm{NMR} ;{ }^{25}$ viscosity-average molecular weight, $\mathrm{M}_{v}, 43,500$ ) was purchased from Polymar Ciência e Nutrição S/A (Fortaleza, Brazil). $10 \mathrm{~g}$ of chitosan was spread in a Petri dish and placed in a closed desiccator containing a mixture of nitric acid $(112 \mathrm{~mL})$ and $\mathrm{H}_{3} \mathrm{PO}_{4}(28 \mathrm{~mL})$. To the acid mixture, $\mathrm{NaNO}_{2}(2 \mathrm{~g})$ was directly added and an immediate formation of reddish brown gases occurred. The chitosan sample in the chamber was allowed to react with the gases for 6 hours, where the obtained aldehyde-functionalized chitosan (hereinafter referred as ALDCHIT) was removed, washed with acetone $(5 \times 100 \mathrm{~mL})$ under stirring, and then dried under vacuum at $50{ }^{\circ} \mathrm{C}$ for 24 hours. The dried powder $(\sim 10.5 \mathrm{~g})$ was then put in distilled water $(200 \mathrm{~mL})$, stirred for $24 \mathrm{~h}$ and then centrifuged in order to separate the impurities and insoluble fraction. The supernatant (soluble fraction) was lyophilized and the powder so obtained was stored in a sealed plastic jar until use. 


\section{Preparation of the ALDCHIT semi-solid hydrogels}

The semi-solid hydrogels were prepared by dissolving ALDCHIT at a concentration of $4 \%(w / w)$ in water, followed by the addition of oxidized dextrose solution (OXDEXT), as the crosslinker agent, at different mole ratios in respect to ALDCHIT. After stirring at room temperature, the semi-solid hydrogels were obtained and referred to as ALDCHIT:OXDEXT 10:1, 10:2 and 10:4, meaning that for 10 moles of ALDCHIT, 1, 2 or 4 moles of OXDEXT were used, respectively. Despite the fact that different volumes of the OXDEXT solution were added to the ALDCHIT hydrogel precursor solution, the final concentration of ALDCHIT (4\%) was kept the same for all samples.

\section{Preparation of oxidized dextrose (OXDEXT)}

The oxidation of dextrose was performed following the method described by Cortesi et al. ${ }^{26}$ where $3 \mathrm{~g}$ of dextrose was dissolved in $15 \mathrm{~mL}$ of distilled water and an excess of sodium periodate $(3 \mathrm{x}$ the number of moles of dextrose) was slowly added under stirring at room temperature in the dark. After 24 hours, a solution of barium acetate was added with the purpose of removing the excess of unreacted iodate. After the addition of barium acetate, a white precipitate was obtained due to the formation of $\mathrm{Ba}_{5}\left(\mathrm{IO}_{6}\right)_{2}$. The precipitate was removed by centrifugation. This process continued until no precipitation was observed. The excess of barium ions was eliminated by the addition of sodium sulfate followed by centrifugation. The obtained oxidized dextrose solution (referred to as OXDEXT) was kept in closed glass vials at $5^{\circ} \mathrm{C}$.

According to Cortesi et al.,${ }^{26}$ the treatment of mono- and disaccharides with periodate leads to the formation of dialdehydes and a complete oxidation is achieved by using an excess of periodate and under the described conditions.

The colorimetric method was used to determine the concentration of dextrose in the solution, as described by DuBois et al. ${ }^{27}$ After proper dilution, $2 \mathrm{~mL}$ of the OXDEXT solution was pipetted to a 10 $\mathrm{mL}$ volumetric flask and $1 \mathrm{~mL}$ of $5 \%$ phenol solution was added. Next, $5 \mathrm{~mL}$ of concentrated $\mathrm{H}_{2} \mathrm{SO}_{4}$ was added and the mixture was allowed to stand for $10 \mathrm{~min}$, where a yellowish color appeared. The volume was topped up with distilled water and the solution was placed in a water bath at $20{ }^{\circ} \mathrm{C}$, before reading was taken at $480 \mathrm{~nm}$ using a HP 8453 UV-visible spectrophotometer (Hewlett-Packard Company, Waldbronm, Germany) equipped with a Hewlett-Packard HP ChemStation software. The concentration of OXDEXT in the solution was determined using a calibration curve prepared using different concentrations of dextrose following the same procedure.

\section{Rheological measurements}

The oscillatory viscoelastic measurements were performed using a rheometer (Haake RheoStress 1 Rotational Rheometer, Haake MessTechnik GmbH. Co., Karlsruhe, Germany), equipped with a $35 \mathrm{~mm}$ parallel-plate and a RheoWin Pro Data Manager (version 2.96) data acquisition software. The viscoelastic parameters determined by the oscillatory measurements were the storage or elastic modulus (G') and the loss or viscous modulus (G").

In order to investigate the influence of the mole ratio between ALDCHIT and OXDEXT on the gelation time $\left(\mathrm{t}_{\mathrm{gel}}\right)$, oscillatory time sweep tests were performed with the ALDCHIT:OXDEXT gel precursor solutions, where $1 \mathrm{~g}$ of each solution, obtained immediately after mixing ALDCHIT with OXDEXT, was placed between the parallel plates of the rheometer. G' and G' were monitored as a function of time at a constant frequency $(1 \mathrm{~Hz})$ and constant shear rate $(60 \mathrm{~Pa})$ at $25{ }^{\circ} \mathrm{C}$. The shear rate was previously verified to be in the linear viscoelastic range.

In addition, the rheological properties were determined with the ALDCHIT:OXDEXT semi-solid hydrogels. The experiments were performed at $37^{\circ} \mathrm{C}$, where $\sim 1 \mathrm{~g}$ of each ALDCHIT:OXDEXT semi-solid hydrogel was carefully placed over the bottom plate of the rheometer. Next, the top plate was brought down until a distance of $1.0 \mathrm{~mm}$ between the plates was reached. The viscoelastic parameters of the semisolid hydrogels were determined as a function of frequency (f). Thus, frequency sweep tests were conducted from 0.08 to $1.6 \mathrm{~Hz}$ at a constant shear rate $(60 \mathrm{~Pa})$, where the latter was verified to be in the linear viscoelastic range.

\section{Determination of water uptake capacity (WUC)}

To determine the $\mathrm{pH}$-dependent water uptake capacity of the lyophilized hydrogels, an accurately weighed amount of each sample was immersed in citrate buffer ( $\mathrm{pH} 4.2$ ) or phosphate buffer ( $\mathrm{pH} 7.4$ ) at $37{ }^{\circ} \mathrm{C}$. At pre-determined time intervals, swollen samples were removed and the excess of water was blotted from the surface with a filter paper and then weighed. The water uptake capacity (WUC) was determined (in percentage) according to the following equation:

WUC $(\%)=\frac{\text { weight of the wet sample }- \text { weight of the dry sample }}{\text { weight of the dry sample }} \times 100$

\section{Preparation of porous tubular scaffolds}

Porous tubular scaffolds were prepared using the lyophilization method. First, the ALDCHIT:OXDEXT 10:4 semi-solid hydrogel was spread uniformly on the annular space of a hollow cylindrical mold with a centered rod (Figure 1).

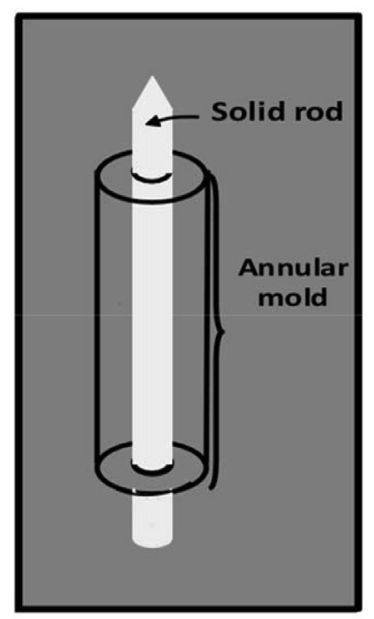

Figure 1. Cilindrical mold used for preparing the tubular scaffolds, where the semi-solid hydrogels were spread on the hollow outer mold

The mold containing the semi-solid hydrogel was left in the vertical position for 12 hours to allow the hydrogel to flow and fill all the gaps that might have been left. Next, the semi-solid ALDCHIT:OXDEXT 10:4 hydrogel was frozen by placing the mold into direct contact with dry ice (temperature of $-78^{\circ} \mathrm{C}$ ). Once the hydrogel was completely frozen, two sets of samples were designed before going through the sublimation step:

Sample 1: the annular/external mold was removed from the assembly while the rod was kept in place. This allowed the exposure of only the outer surface of the hydrogels during the sublimation step.

Sample 2: the center rod was removed but the annular mold was 
left in place, allowing only the hydrogel's inner surface to be exposed, while keeping the outer surface covered by the annular mold.

The assembly containing the frozen hydrogel with either the inner or the outer surface exposed was then taken to the freeze-drier and lyophilized for 48 hours. The obtained porous tubular scaffolds were kept in sealed glass vials until use.

\section{Scanning electron microscope}

The morphologies of the tubular scaffolds were obtained by scanning electron microscope (SEM), where the samples were mounted on stubs and sputter coated using K550 Emitech Sputter Coater (Emitech Ltd, Kent, England), then imaged at $3.0 \mathrm{kV}$ on a Hitachi S-4800 field emission scanning electron microscope (Hitachi High-Technologies Corporation, Tokyo, Japan).

\section{RESULTS}

\section{Preparation of the ALDCHIT:OXDEXT semi-solid hydrogels}

In this work, semi-solid hydrogels were prepared by simply dissolving the novel water soluble aldehyde-functionalized chitosan (ALDCHIT) in distilled water at a concentration of $4 \%(w / w)$, without the addition of acids. Since the primary objective of this work is to produce tubular scaffolds for vascular tissue engineering, a more rigid structure is desired, which could withstand the high stresses that these scaffolds are usually submitted during the cell seeding and tissue growth steps. Thus, the ALDCHIT 4\% solution was crosslinked with different amounts of oxidized dextrose (OXDEXT). This crosslinker was used in an attempt to avoid the commonly used dialdehydes and therefore, obtain a more biocompatible hydrogel. The dissolution of ALDCHIT as well as its crosslinkage with OXDEXT occurred at room temperature in a fully water-based environment, free of acids and toxic crosslinkers.

ALDCHIT could easily dissolve in water, forming a clear viscous solution at $4 \%$ where it gelified upon the addition of OXDEXT. The gelation time and the stiffness of the obtained semi-solid hydrogel were influenced by the ALDCHIT:OXDEXT mole ratio (10:1, 10:2 and 10:4).

\section{Rheological measurements}

The gelation process (sol-gel transition) of any pre-gel solution is characterized by a crossover between G' and G', with the former becoming higher than the latter. In order to verify the influence of the amount of OXDEXT on the gelation time $\left(\mathrm{t}_{\mathrm{gel}}\right)$ of the ALDCHIT 4\% solutions, G' and G" were determined as a function of time immediately after ALDCHIT 4\% and OXDEXT solutions were mixed. Figure 2 shows that initially all samples were purely viscous, with a higher value of the loss modulus, G", compared to the elastic modulus, G'. However, both G' and G" increased as the reaction progressed, with the former increasing much faster than the latter, which is a typical behavior of a crosslinking process. The time taken to reach this cross-over point denotes the gelation time $\left(\mathrm{t}_{\mathrm{gel}}\right)$ and according to Figure 2, as the amount of OXDEXT increased, the $t_{\text {gel }}$ decreased.

Figure 3 shows the gelation time as a function of the mole ratio of ALDCHIT to OXDEXT, where it shows an almost linear decrease in the $t_{\text {gel }}$ with the increase in the amount of crosslinker. Assuming that the formation of these semi-solid hydrogels was governed mainly by the extent of crosslinking of the ALDCHIT chains, the rate of crosslinking should be proportional to the initial OXDEXT concentration in the hydrogel precursor solution.

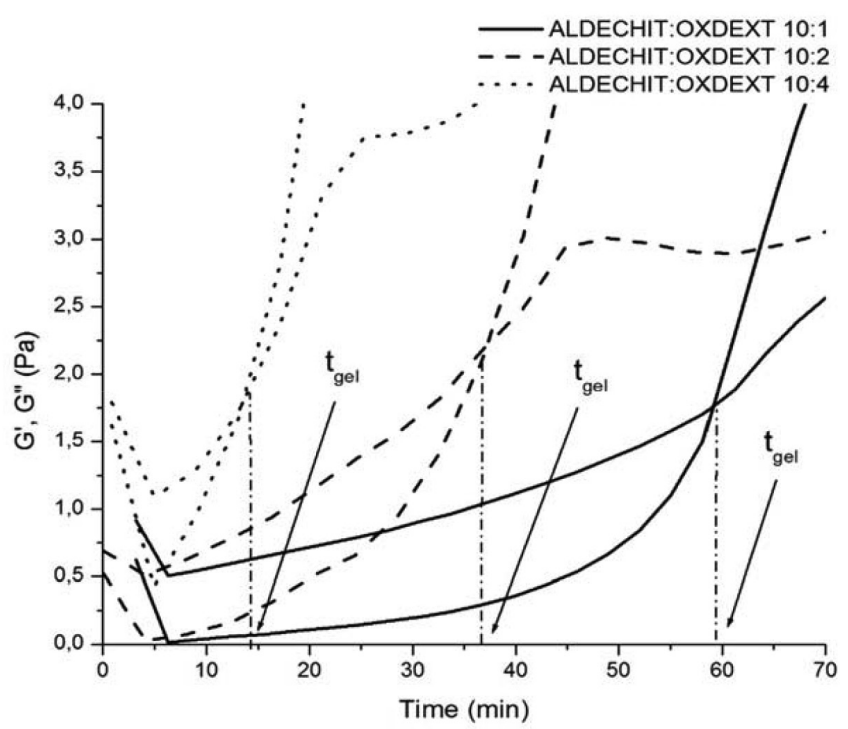

Figure 2. G' and G" crossover of different ALDCHIT:OXDEXT molar ratios (dotted line: 10:4; dashed line: 10:2; solid line: 10:1), showing the influence of the amount of the crosslinking agent on the gelation time $\left(t_{\text {gel }}\right)$

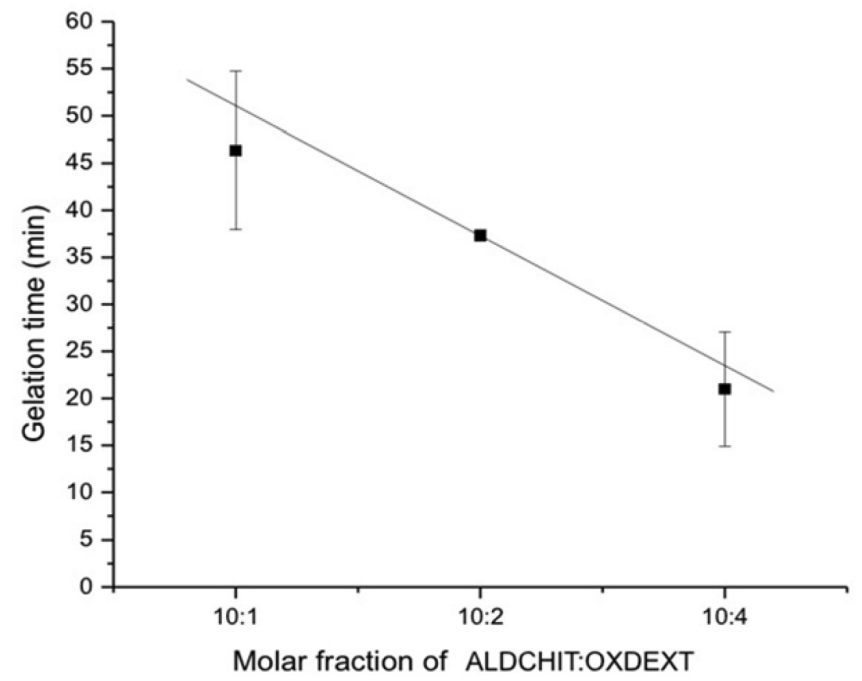

Figure 3. Relationship between the mole ratio of ALDCHIT:OXDEXT and gelation time $\left(t_{g e l}\right)(n=3$; error bar: standard deviation $)$

On the other hand, Figure 4 shows the change in storage modulus (G') according to the applied shear stress on the ALDCHIT:OXDEXT semi-solid hydrogels. G' increased with increasing shear stress until a maximum value was reached. Then, as the shear stress kept increasing, G' decreased until it almost reached zero, which means that the three-dimensional network of the hydrogel was broken. This yield value is called yield stress and when it is exceeded, the hydrogels flow like liquids. Therefore, at stress values below the yield stress they produce mainly elastic deformations, whereas at stress values above the yield stress, the network is partially ruptured and flow occurs. ${ }^{28}$ The initial increase in G' with the shear stress might be due to an increase in chain entanglement when the hydrogels are submitted to shear stresses. In addition, the shear stress might have increased the mobility of the chains, which possibly allowed for more interactions between ALDCHIT and OXDEXT.

\section{Water uptake capacity (WUC)}

The water uptake of the ALDCHIT:OXDEXT lyophilized 


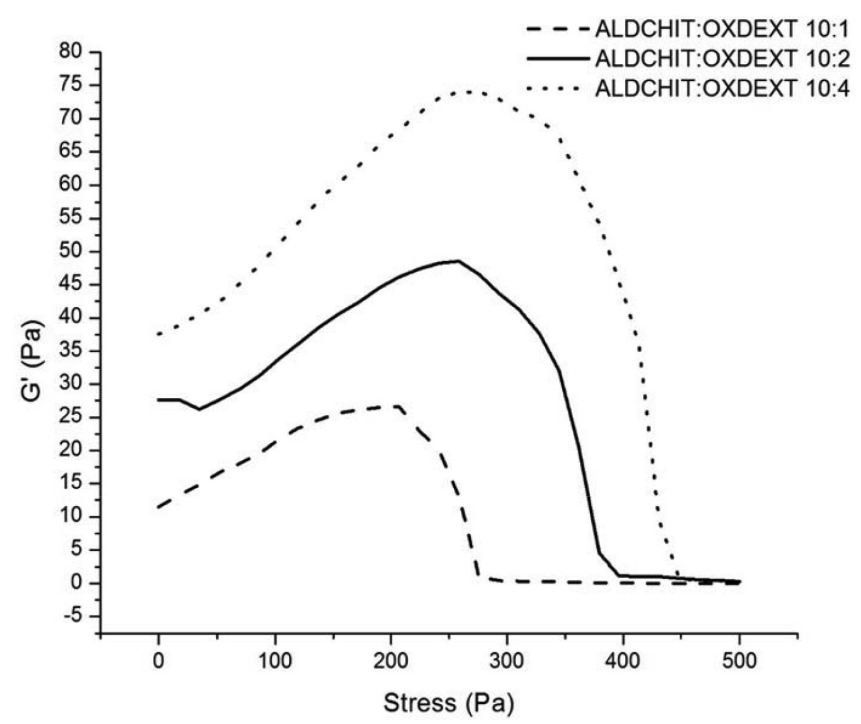

Figure 4. Elastic modulus $\left(G^{\prime}\right)$ versus shear stress for ALDCHIT:OXDEXT hydrogels prepared with different crosslinking density

hydrogels in $\mathrm{pH} 4.2$ and 7.4 at $37{ }^{\circ} \mathrm{C}$ is shown in Figure 5. All the hydrogel samples increased more than $200 \%$ in weight in the first 30 minutes, regardless of the ALDCHIT:OXDEXT mole ratio. Further, the weight of the hydrogels began to decrease as some dissolution occurred, which was probably caused by the hydrolysis of the imine bonds after such a high extent of water uptake. However, it is clear that the water uptake reached a more stable equilibrium with the 10:4 samples at $\mathrm{pH} 7.4$, where no weight loss was observed. The ALDCHIT:OXDEXT 10:4 hydrogels could uptake up to $400 \%$ of its weight in water with no loss of mass during the entire course of the experiment (one week).

\section{Preparation of the ALDCHIT:OXDEXT porous tubular scaffolds}

In this study, since the ALDCHIT:OXDEXT ratio that yielded the most stable hydrogel at pH 7.4 was 10:4 (Figure 5), the use of this hydrogel to prepare scaffolds for tissue engineering was investigated. Thus, tubular scaffolds were prepared from ALDCHIT:OXDEXT 10:4 semi-solid hydrogels using the lyophilization method, where the orientation of the pores was controlled by exposing either the internal or the external surface of the frozen hydrogel during the sublimation step.

Figure 6 shows the inner and outer surfaces of the ALDCHIT:OXDEXT 10:4 tubular scaffolds. The method where the internal rod was left inside the frozen hydrogel but the outer mold was removed, originated a scaffold with a non-porous lumen (Figure 6A), but with a porous outer surface, where the pore sizes ranged from 1 to $5 \mu \mathrm{m}$ (Figure 6B). The rough inner surface of this hollow tube could have been originated from the irregular surface of the rod. On the other hand, the sample where the internal rod was removed and the outer mold was kept in place during the sublimation process shows a very small amount of pores on the outer surface (Figure 6C), but a much higher amount of pores in the lumen (Figure 6D). The porous in the inner surface appear to have a high level of interconnectivity.

Figure 7 shows higher magnification pictures of the inner surface of the sample obtained through lyophilization of the frozen hydrogel without the rod, but with the outer mold, where it seems that the pores are interconnected (Figure 7B), with pore sizes ranging from 50 to $150 \mu \mathrm{m}$.
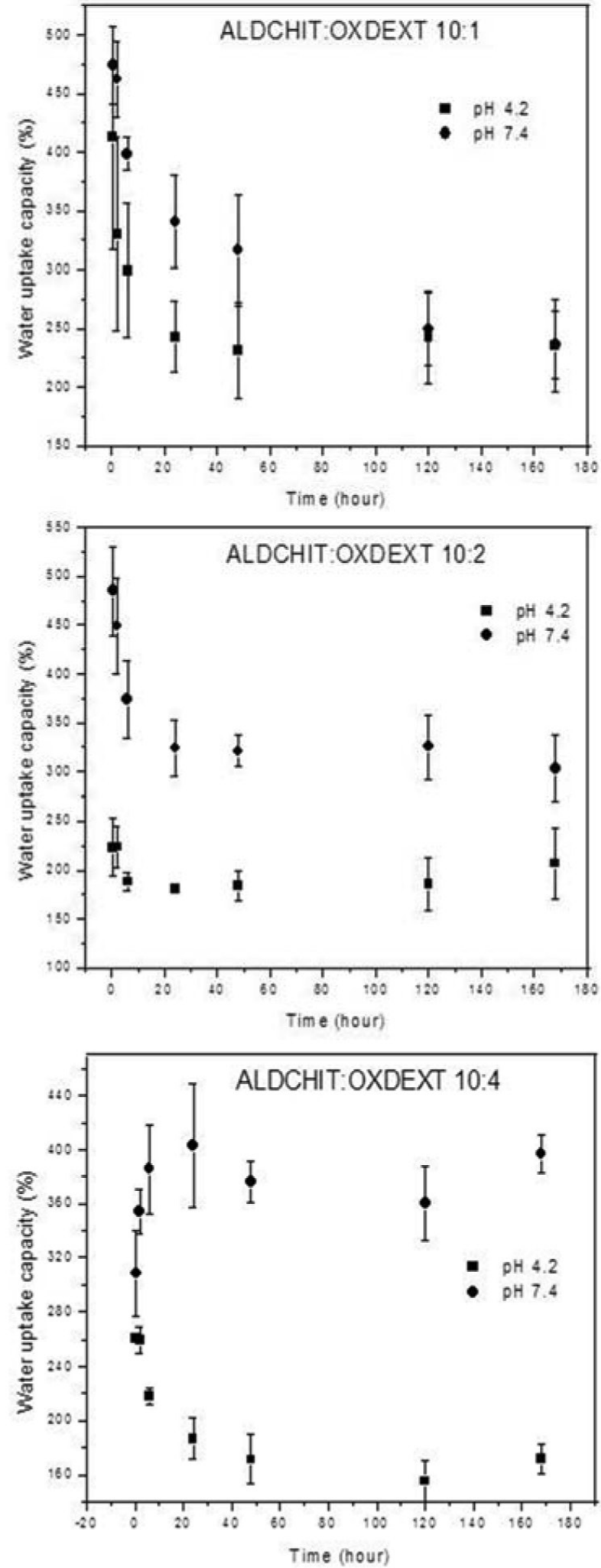

Figure 5. Comparison of the water uptake capacity of the ALDCHIT:OXDEXT freeze-dried hydrogels in $p H 4.2$ and 7.4 buffer solutions. $n=3$; error bar: standard deviation

\section{DISCUSSION}

The wide array of tissue engineering applications is founded upon the use of polymer scaffolds, which serve to support, reinforce and organize the regenerating tissue. ${ }^{29}$ Hydrogels have become attractive 

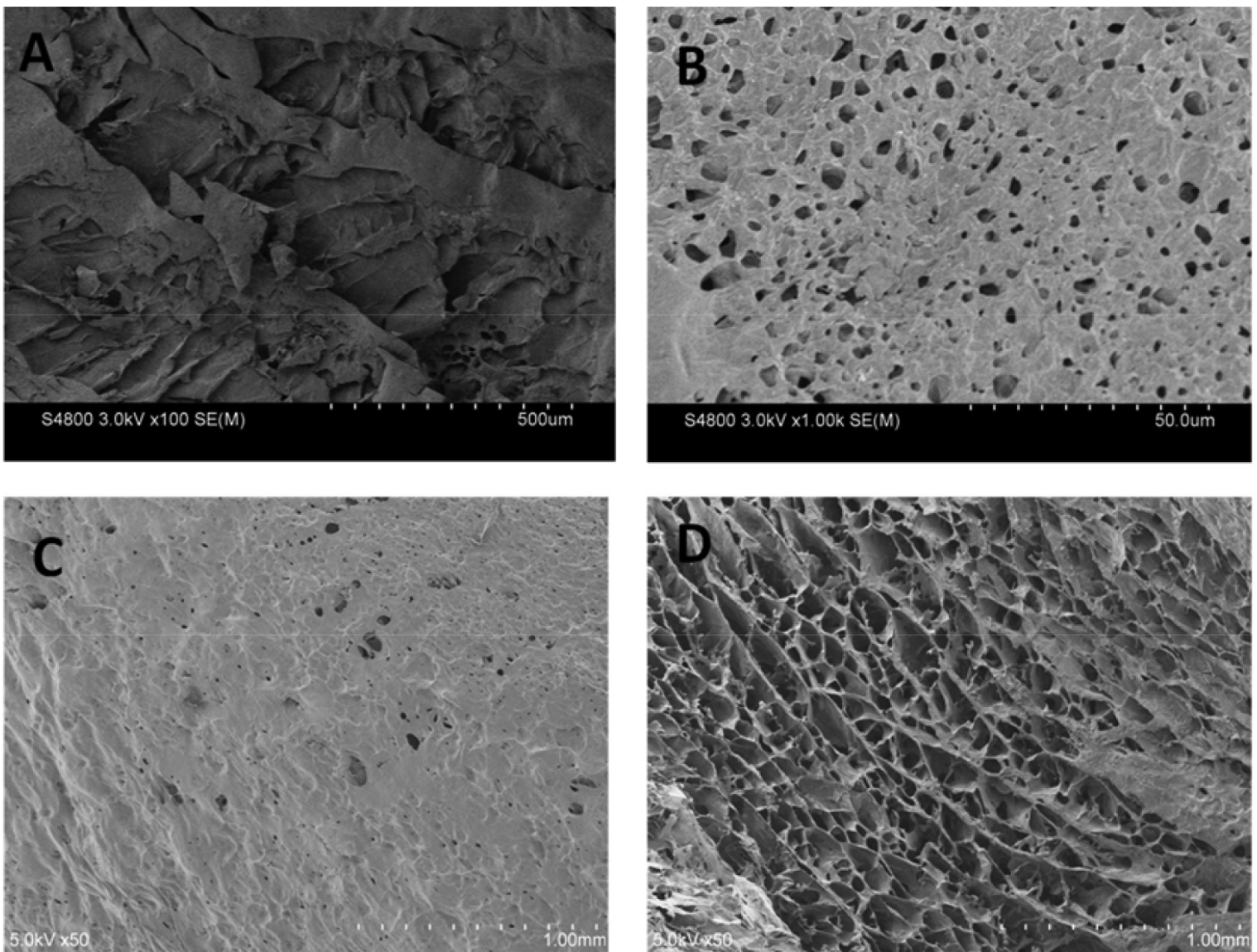

Figure 6. SEM pictures of tubular scaffolds, prepared through the lyophilization of the ALDCHIT:OXDEXT 10:4 semi-solid hydrogels with the center rod and without the outer annular mold (inner surface: A; outer surface: B) and without the rod and with the outer annular mold (outer surface: C; inner surface: D)
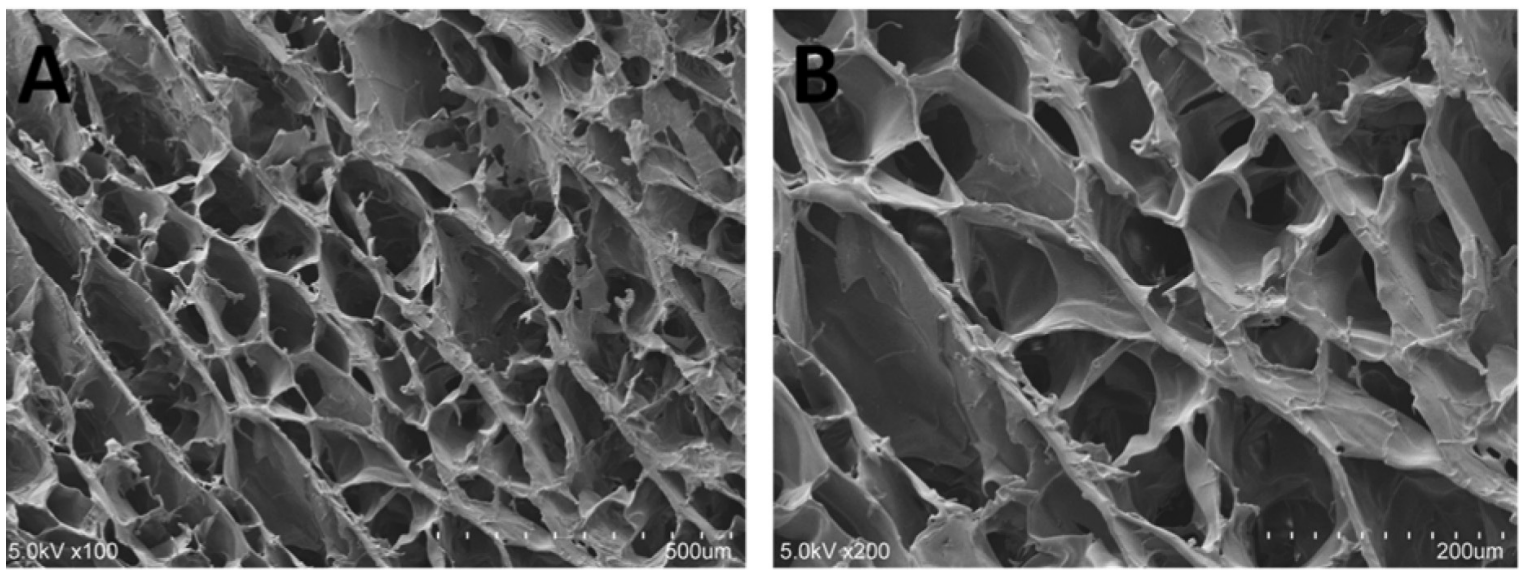

Figure 7. SEM pictures of the pores in the inner surface of the ALDCHIT:OXDEXT 10:4 tubular scaffolds prepared without the center rod and with the outer mold. A: 100x; B: $200 x$

for tissue engineering applications, where they have been used as scaffolds for repairing and regenerating a wide variety of tissues and organs. ${ }^{16}$ Hydrogels derived from naturally occurring polymers have the advantage of mimicking many features of extracellular matrix and therefore, have the potential to direct the migration, growth and organization of cells during tissue formation. ${ }^{30}$ Due to their low immunogenic activity, controlled biodegradability and porous structure, chitosan scaffolds are promising materials for the design of tissue engineered systems. ${ }^{31}$

Chitosan derivatives containing aldehyde functions (ALDCHIT) have been successfully prepared by our group by reacting chitosan with nitrous oxide gases at solid state. In this method, chitosan undergoes deaminative cleavage of the 1,4 glycosidic bond, producing 2,5-anhydro-D-mannose as the reducing end (Figure 8).

Besides being water soluble, this aldehyde-functionalized chitosan could form semi-solid hydrogels by simply dispersing it in water, at proper concentration, without the addition of acids. ${ }^{23}$ Further characterization of the lyophilized hydrogels revealed a highly porous structure with interconnected pores, which was evidenced by their high water uptake capacity. ${ }^{24}$

In this current study, with the purpose of preparing a more rigid structure that could withstand the high stresses that these scaffolds are usually submitted during the cell seeding and tissue growth steps, ALDCHIT was crosslinked using different amounts of a crosslinking 

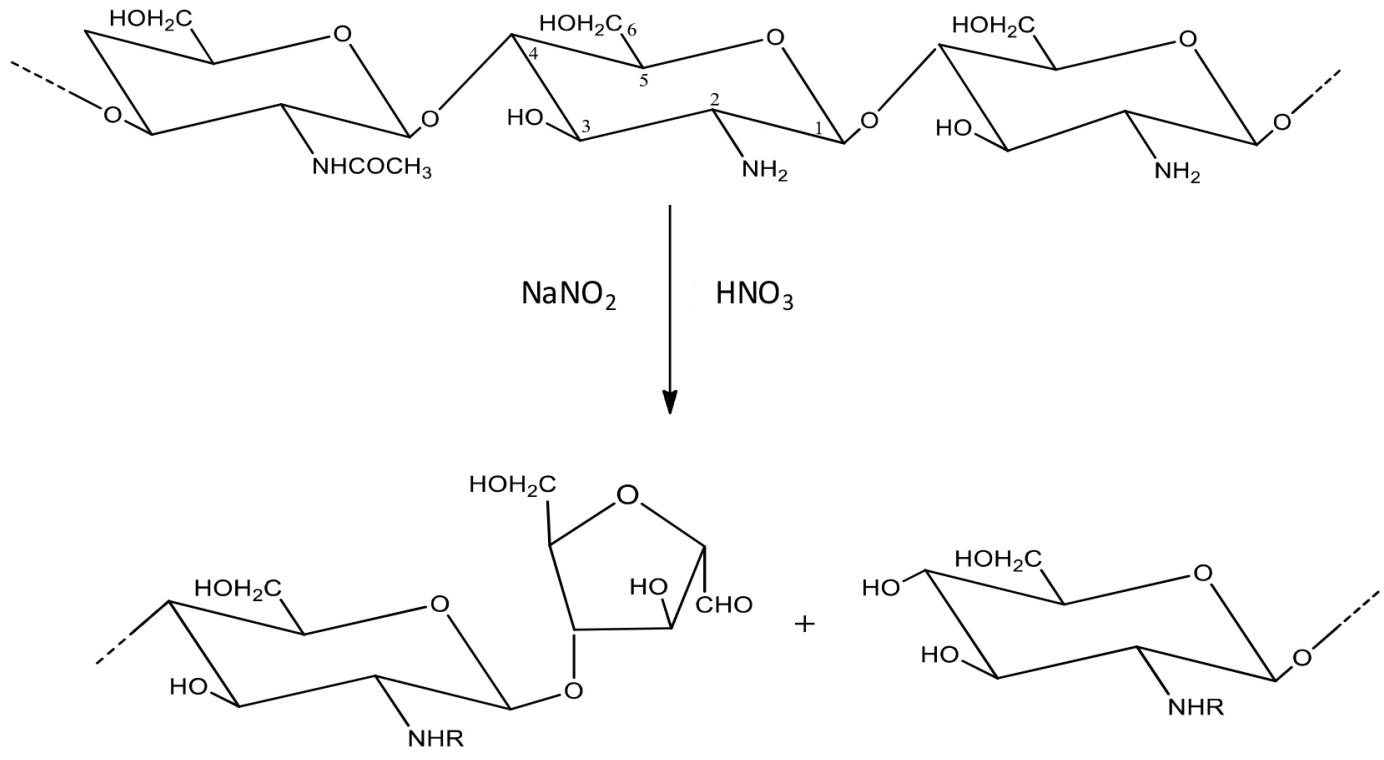

Figure 8. Reaction of chitosan with nitrous oxide gases produced from the reaction of $\mathrm{NaNO}_{2}$ and $\mathrm{HNO}_{3}$ forming the chitosan derivative with the 2,5-anhydroD-mannose at the reducing end

agent. On the other hand, Madihally and $\mathrm{Matthew}^{29}$ have prepared chitosan porous tubular scaffolds without any external crosslinker by just lyophilizing a $2 \%$ chitosan solution in annular molds. The obtained tubular scaffolds, which were stabilized in either $\mathrm{NaOH}(0.1$ $\mathrm{N}$ ) or in ethanol series, showed higher porosity and interconnected pores, but due to the fact that no crosslinkage took place, poor physical and mechanical stability of such constructs became a concern during the washing process and even during the cell seeding/expansion in aqueous medium. ${ }^{29}$

Glutaraldehyde is usually the crosslinker of choice for preparing a more stable chitosan hydrogel/scaffold. For instance, chitosan crosslinked with glutaraldehyde or oxidized dextran has been investigated as a potential scaffold in tissue engineering for cartilage regeneration. ${ }^{16}$ However, considering that the chitosan scaffold will be degraded once implanted into the body, glutaraldehyde will be prone to leach out, which would increase the likelihood of toxicity. ${ }^{30}$

Therefore, in order to prepare a more biocompatible scaffold, we decided to use oxidized dextrose (OXIDEXT) as the crosslinking agent. Cortesi et al. ${ }^{26}$ reported the crosslinking of gelatin with oxidized sugars, where microspheres and disks were prepared by this method. Similarly, Wang and $\mathrm{Hon}^{32}$ and Li et al. ${ }^{21}$ used oxidized sugars to prepare crosslinked chitosan-PEG and $N$-alkylated chitosan membranes, respectively. Pourjavadi et al. ${ }^{33}$ designed hydrogels based on chitosan using tetraaldehyde molecule as the crosslinking agent, obtained from periodate oxidation of sucrose, where the hydrogels showed a reasonable swelling $\mathrm{pH}$ sensitivity and an enhanced biocompatiblity.

According to Figure 2, $\mathrm{t}_{\text {gel }}$ was inversely proportional to the amount of OXDEXT added. This indicates that increasing the crosslinking reduces the time needed to form the gel, which is probably due to the increasing number of the imine linkages (Schiff base). In addition, the maximum G' reached, which is related to the elasticity and solid-like behavior of the hydrogel, varied according to the amount of OXDEXT in respect to ALDCHIT (Figure 4). According to Singh, ${ }^{1}$ the chemical crosslinking seems to be the best approach to improve the strength of hydrogels. In fact, a higher crosslinker/ monomer ratio seems to enhance the strength and elasticity of the ALDCHIT:OXDEXT hydrogel, as evidenced by the higher complex modulus, G' (Figure 4). In addition, Hoare and Kohane ${ }^{18}$ stated that the extent of crosslinkage can control variables such as gelation time, mechanical strength, network pore size, degradation time and degree of swelling of hydrogels.

In fact, the ALDCHIT:OXDEXT hydrogel prepared with the highest crosslinker/monomer ratio (ALDCHIT:OXDEXT 10:4) was the most stable sample at pH 7.4 (Figure 5). Since the pKa of chitosan is around 6.5, it is well known that chitosan hydrogels deprotonate at $\mathrm{pH}$ values above $7.1,17,18,23$ Therefore, besides the higher amount of crosslinker in the ALDCHIT:OXDEXT 10:4 hydrogel, it seems that at $\mathrm{pH} 7.4$ the $-\mathrm{NH}_{3}{ }^{+}$is deprotonated and the imine linkages are favored, which suggests that the higher numbers of imine bonds in the 10:4 sample and the higher stability of these bonds at higher $\mathrm{pH}$, could explain why the ALDCHIT:OXDEXT 10:4 hydrogel at pH 7.4 was the most stable one. The reasonably high WUC of these hydrogels might indicate that they have a high level or porosity.

Therefore, the potential use of ALDCHIT:OXDEXT 10:4 hydrogels to prepare scaffolds for vascular tissue engineering was investigated, where tubular constructs were prepared by lyophilization. In this method, the water inside the hydrogels is frozen and then sublimated, which eventually creates pores. In this study, the orientation of the pores was controlled by exposing either the internal or the external surface of the frozen hydrogel during the sublimation step.

According to Harley et al., ${ }^{34}$ an ideal porous tubular scaffold should display a gradient in porosity and pore size along the tube radius, with a porous lumen and a cell-impermeable outer surface. In addition, Song et al. ${ }^{35}$ showed that the efficiency of smooth muscle cells (SMC) seeding in porous tubular poly(trimethylene carbonate) (PTMC) scaffolds increased from less than 10\% to $43 \%$ after coating its external surface with a PTMC layer while still keeping the inner porous structure.

SMC are usually seeded by perfusion of a cell suspension from the lumen through the wall of a tubular scaffold, where the absence of pores on the outer surface leads to higher cell retentions. Moreover, in order to have a better diffusion of nutrients and waste products, as well as a more efficient cell proliferation, a more open and interconnected pore structure is preferred. ${ }^{35}$ Figure 7 shows higher magnification pictures of the inner surface of the ALDCHIT:OXDEXT tubular scaffolds obtained through the lyophilization of the frozen hydrogel without the rod, but with the outer mold, just to emphasize the high level of pore interconnectivity, with pore sizes ranging from 50 to $150 \mu \mathrm{m}$. The orientation, interconnection and size of the pores in 
biomedical scaffolds have been shown to control the rate and depth of cellular ingrowth in vivo. In fact, Zeltinger et al. ${ }^{36}$ showed that vascular SMC displayed a better proliferation and matrix deposition in scaffolds with pore sizes ranging from 63 to $150 \mu \mathrm{m}$. Similarly, Park et al. ${ }^{37}$ showed that SMC proliferation was the highest in a poly(L-lactide-co- $\varepsilon$-caprolactone) (PLCL) scaffold with pore sizes between 50 and $100 \mu \mathrm{m}$.

Therefore, the presence of interconnected pores on the inner surface, with pore sizes ranging from 50 to $150 \mu \mathrm{m}$, in addition to the less porous outer surface, make the ALDCHIT:OXDEXT hollow tube, prepared by lyophilization of the hydrogels without the rod and with the outer jacket, a promising candidate as a scaffold for vascular tissue engineering.

\section{CONCLUSIONS}

In conclusion, the ALDCHIT:OXDEXT semi-solid hydrogels could successfully originate tubular scaffolds using the lyophilization method, where the orientation of the pores could be controlled by exposing either the internal or the external surface of the frozen hydrogel during the sublimation step. Since the hydrogels were obtained from a water-soluble chitosan derivative (ALDCHIT) using a biocompatible crosslinking agent (OXDEXT), we strongly believe that the aqueous environment and the lack of toxic additives might contribute to an enhanced biocompatibility of these constructs. Finally, the adhesion and proliferation of vascular smooth muscle cells on the ALDCHIT:OXDEXT tubular scaffolds are currently being investigated in our lab.

\section{ACKNOWLEDGMENTS}

The authors would like to thank CAPES/Fulbright exchange program for financial support.

\section{REFERENCES}

1. Singh, A.; Bull. Mater Sci. 2006, 29, 233.

2. Ravi Kumar, M. N. V.; React. Funct. Polym. 2000, 46, 1.

3. Vodna, L.; Bubenikova, S.; Lacik, I.; Chorvat Jr., D.; Bakos, D.; Macromol. Biosci. 2007, 7, 629.

4. Zheng, J. N.; Xie, H. G.; Yu, W. T.; Liu, X. D.; Xie, W. Y.; Zhu, J.; Ma, X. J.; Langmuir 2010, 26, 17156.

5. Pashkunova-Martic, I.; Kremser, C.; Galanski, M.; Arion, V.; Debbage, P.; Jaschke, W.; Keppler, B.; Mol. Imaging Biol. 2011, 13, 16.

6. Chatterjee, S.; Lee, M. W.; Woo, S. H.; Bioresour. Technol. 2010, 101, 1800 .

7. Chatterjee, S.; Lee, D. S.; Lee, M. W.; Woo, S. H.; Bioresour. Technol. 2010, 101, 4315 .

8. Chatterjee, S.; Chatterjee, T.; Lim, S. R.; Woo, S. H.; Bioresour. Technol. 2011, 102, 4402.

9. Horio, T.; Ishihara, M.; Fujita, M.; Kishimoto, S.; Kanatani, Y.; Ishizuka, T.; Nogami, Y.; Nakamura, S.; Tanaka, Y.; Morimoto, Y.; Maehara, T.; Artif. Organs 2010, 34, 342.
10. Ribeiro, M. P.; Espiga, A.; Silva, D.; Baptista, P.; Henriques, J.; Ferreira, C.; Silva, J. C.; Borges, J. P.; Pires, E.; Chaves, P.; Correia, I. J.; Wound Repair and Regeneration 2009, 17, 817.

11. Zhang, Y.; Ji, C.; Anal. Chem. 2010, 82, 5275.

12. Leipzig, N. D.; Wylie, R. G.; Kim, H.; Shoichet, M. S.; Biomaterials 2011, 32, 57.

13. Madhumathi, K.; Shalumon, K. T.; Rani, V. V.; Tamura, H.; Furuike, T.; Selvamurugan, N.; Nair, S. V.; Jayakumar, R.; Int. J. Biol. Macromol. 2009, 45, 12.

14. Hong, Y.; Gong, Y.; Gao, C.; Shen, J.; J. Biomed. Mater. Res. A 2008, 85,628 .

15. Berger, J.; Reist, M.; Mayer, J. M.; Felt, O.; Gurny, R.; Eur. J. Pharm. Biopharm. 2004, 57, 35.

16. Hoffmann, B.; Seitz, D.; Mencke, A.; Kokott, A.; Ziegler, G.; J. Mater. Sci. Mater. Med. 2009, 20, 1495.

17. Knaul, J. Z.; Hudson, S. M.; Creber, K. A. M.; J. Polym. Sci., Part B: Polym. Phys. 1999, 37, 1079.

18. Hoare, T. R.; Kohane, D. S.; Polymer 2008, 49, 1993.

19. Muzzarelli, R. A. A.; Carbohydr. Polym. 2009, 77, 1.

20. Baran, E. T.; Mano, J. F.; Reis, R. L.; J. Mater. Sci. Mater. Med. 2004, 15,759 .

21. Li, F.; Liu, W. G.; Yao, K. D.; Biomaterials 2002, 23, 343.

22. Moura, M. J.; Biomacromolecules 2007, 8, 3823.

23. Azevedo, E. P.; Santhana Mariappan, S. V.; Kumar, V.; Carbohydr. Polym. 2012, 87, 1925 .

24. Azevedo, E. P.; Kumar, V.; Carbohydr. Polym. 2012, 90, 894.

25. Lavertu, M.; Xia, Z.; Serreqi, A. N.; Berrada, M.; Rodrigues, A.; Wang, D.; Buschmann, M. D.; Gupta, A.; J. Pharm. Biomed. Anal. 2003, 32, 1149.

26. Cortesi, R.; Nastruzzi, C.; Davis, S. S.; Biomaterials 1998, 19, 1641.

27. DuBois, M.; Gilles, K. A.; Hamilton, J. K.; Rebers, P. A.; Smith, F.; Anal. Chem. 1956, 28, 350.

28. Schott, H. In Remington: The Science and Practice of Pharmacy; Gennaro, A. R., ed.; Lippincott Williams \& Wilkins: Philadelphia, 2000, cap 7.

29. Madihally, S. V.; Matthew, H. W.; Biomaterials 1999, 20, 1133.

30. Balakrishnan, B.; Jayakrishnan, A.; Biomaterials 2005, 26, 3941.

31. Aranaz, I.; Mengibar, M.; Harris, R.; Panos, I.; Miralles, B.; Acosta, N.; Galed, G.; Heras, A.; Curr. Chem. Biol. 2009, 3, 203.

32. Wang, J. W.; Hon, M. H.; J. Mater. Sci. Mater. Med. 2003, 14, 1079

33. Pourjavadi, A.; Aghajani, V.; Ghasemzadeh, H.; J. Appl. Polym. Sci. 2008, 109, 2648.

34. Harley, B. A.; Hastings, A. Z.; Yannas, I. V.; Sannino, A.; Biomaterials 2006, 27, 866

35. Song, Y.; Wennink, J. W. H.; Kamphuis, M. M. J.; Vermes, I.; Poot, A. A.; Feijen, J.; Grijpma, D. W.; J. Biomed. Mater. Res. A 2010, 95A, 440.

36. Zeltinger, J.; Sherwood, J. K.; Graham, D. A.; Mueller, R.; Griffith, L. G.; Tissue. Eng. 2001, 7, 557.

37. Park, I. S.; Kim, S. H.; Kim, I. H.; Kim, S. H.; J. Biomater. Sci., Polym. Ed. 2009, 20, 1645. 